\title{
Service Value Broker Patterns: An Empirical Collection and Analysis
}

\author{
Yucong Duan \\ College of information science and technology, Hainan University, China \\ Email:duanyucong@hotmail.com \\ Keman Huang* \\ Department of Automation, Tsinghua University, China \\ Email:victoryhkm@gmail.com \\ Dan Chen \\ College of information science and technology, Hainan University, China \\ Email:chendan@hainu.edu.cn \\ Yongzhi Wang \\ Florida International University, Miami, USA \\ Email:ywang032@cis.fiu.edu \\ Ajay Kattepur \\ ARLES group, INRIA Paris-Rocquencourt, France \\ Email:ajay.kattepur@inria.fr \\ Wencai Du* \\ College of information science and technology, Hainan University, China \\ Email:wencai@hainu.edu.cn \\ Received 22 February 2013 \\ Accepted 19 November 2013
}

\begin{abstract}
The service value broker(SVB) pattern integrates business modeling, knowledge management and economic anal- ysis with relieved complexity, enhanced reusability and effi- ciency,etc. The study of SVB is an emerging interdisciplinary subject which will help to promote the reuse of knowledge, strategy and experience in service based designs and solutions. In this paper, we focus on enumerating collected SVBs empir- ically with initial analysis on their composition manners. The results from this paper will play a dominating role in fueling a coming E-service Economics era.
\end{abstract}

Keywords: service value, design pattern, business modeling, broker, economics

Published by Atlantis Press

Copyright: the authors 


\section{Introduction}

This paper is an extension of [14]. Software design patterns [18] have been proved proposed and verified successfully in the modeling processes of multiple technical domains. However for modeling for modeling service oriented computing (SOC) applications, design patterns have to be adapted according to value of Quality of Service (QoS) or business contractual aspects. We refer to this as the Service Value Broker(SVB) [10], [13] pattern. $S V B$ has already been proposed for cloud service brokerage [29] which we foresee as an important characteristics of the optimization of the E-Service composition of [22] E-Service Economics.

To clarify the novelty of $S V B$ in contrast with traditional design patterns, we study the increments provided to various concepts of value from a service perspective [19]:

i. Functional value - The domain in question relates $\mathbb{D}_{F}$ : Data Quality that improves the output functional data of the service. An example would be compositions with superior services that can provide better quality output. It is further distinguished as:

a. implementation function value - related to content data and

b. control function value - related to control information.

ii. QoS value - that improves performance from domains $\mathbb{D}_{Q}$ : latency, availability, throughput and other domains[23]. Each domain may have aggregation and composition rules specified.

iii. Security value - that determines the necessary condi- tions of a business transaction such as $\mathbb{D}_{S}$ : location, IP address, legal issues [10] in service contracts [9].

iv. Business value - that directly related to the increase/decrease of the $\mathbb{D}_{B}$ : monetary, non-monetary value [10] of the goods/services of a transaction.

v. Value added $-\mathbb{D}_{V}$ that can come from service reuse [11] in terms of either directly plugging in a service or identifying novel usage with service composition [22].

Traditional design patterns are usually introduced for knowledge and technical reuse which is fully related to 1 and 2, and partially related to $3 . S V B$ is fully compatible with the advantage of traditional design value and at the same time covers all five listed items 1-5. SVB directly centers service value implementation and service value optimization. To the best of our knowledge, there is little work available in this field, we work towards contributing to the target of laying down a foundation of models of $S V B$ pattern through demon- strating a set of $S V B$ patterns. A simplified formulation of the difference between constructing a traditional broker and a $S V B$ is as follows:

i. Quality driven - For constructing a traditional broker, a composing service is chosen based on the order of the quality of its functionality. And for a set of composing services, the priority is in ratio to:

$\sum_{\text {integration }}(\text { quality })_{i \ldots n}$.

ii. Price/quality driven - For constructing a SVB, a composing service is chosen based on the order of the price/quality of its functionality. And for a set of composing services, the priority is in ratio to:

$$
\sum_{\text {integration }}(\text { price/ quality })_{i \ldots n}+\Delta_{\text {valueadded }} .
$$

The rest of the paper is organized as follows: Section II demonstrates $S V B$ patterns with explanations on the scenar- ios. Section III presents the composition and organization of $S V B$ patterns. Section IV models a twolevel E-contract based implementation framework. Section V explains the case for the service contract broker. Section VI provides a simulation to demonstrate the $S V B$ pattern in use. This is followed by related work in Section VII and conclusions with future directions in Section VIII.

\section{Service Value Broker Patterns: Scenarios and Brokers}

SVB and DSVB are required for constructing E-Service transaction scenarios. Therefore, we we can assume the existence of E-Contracts among service providers or stakeholder, which are wrote with shared language[12] and backed by administrative and juridical power where 
the restrictions must be met for any qualified composition and matchmaking [9]. The following discussion is based on the premise that a service or a group of services which choose to play the roles of SVB/DSVB will be rewarded with a profit not less than the price of fulfilling a normal business transaction based on E-Contracts. We enrich the basic scenarios in [10] which might happen as follows:

We denote the contract on the source end of an exchange as CS, the contract on the target end of an exchange as CT, the input of SVB/DSVB contract as iSVB and the output of a SVB/DSVB contract as oSVB. There is no requirement on that the iSVB and $O S V B$ are with the same service since that chained subcontract relationship might happen and also the integration of a parallel set of SVB/DSVB is allowed. We propose to demonstrate the brokerage within Cloud based on the three-layer architecture of SaaS, PaaS, and IaaS. Fig. 1 demonstrates the brokers in relationship with the three layer Cloud architecture.

i. Location $\left(L C \in \mathbb{D}_{S}\right)$ : requests are restricted to be "requested within France" while the customer want to visit "from Italy".

Problem: $\left.L C\right|_{C S} !=\left.L C\right|_{C T}$

$S V S=\left(0, \delta\left(\left.L C\right|_{C T},\left.L C\right|_{C S}\right)\right)$ with $\delta$ representing a change.

Location broker: a service which " accepts requests from Italy" and located in France has the possibility of playing the broker.

Solution: $\left(\left.L C\right|_{C S}=\left.L C\right|_{i S V B}\right) A N D\left(\left.L C\right|_{o S V B}=\right.$ $\left.L C\right|_{C T}$ )

ii. Available time $\left(A T \in \mathbb{D}_{Q}\right)$ : requests are restricted to be "requested from 8:00 AM to 5:00 PM at Italy Time" while the customer want to visit "during 8:00 $\mathrm{AM}$ to 5:00 PM at China Time".

Problem: $\left.A T($ high $)\right|_{C S}<\left.A T($ low $)\right|_{C T}$

$S V S=\left(0, \delta\left(\left.A T\right|_{C T},\left.A T\right|_{C S}\right)\right)$

Available time broker: if the requests/responses from communication services are independent data requests or computation requests, a service which can store the requests/reponses and the states of other services and delay the triggering of the requests for a period of 7 hours can play the broker.

Solution: $\left(A T\right.$ (high) $\left.\right|_{C S}>A T$ (low) $\left.\left.\right|_{i S V B}\right) A N D$
(AT (high) $\left.\right|_{o S V B}>\left.A T($ low $\left.)\right|_{C T}\right)$

iii. Request amount $\left(R A \in \mathbb{D}_{Q}\right)$ : requests are restricted to "600 requests a day" while customers want to " 10000 successful visits /daily".

Problem: $\left.R A\right|_{C S}<\left.R A\right|_{C T}$ $S V S=\left(0, \delta\left(\left.R A\right|_{C T},\left.R A\right|_{C S}\right)\right)$

Request amount broker: a service or service group which owns or runs multiple agents has the possibility to represent the original service request side to convey the requests and responses can play the broker.

Solution: $\sum\left(\left.R A\right|_{C S}=\left.R A\right|_{i S V B}\right) A N D\left(\left.R A\right|_{o S V B}\right.$ $\left.>=\left.R A\right|_{C T}\right)$

iv. Network traffic $\left(N T \in \mathbb{D}_{Q}\right)$ : requests are limited to "band width of $50 \mathrm{M} / \mathrm{min}$ " while customers request " $100 \mathrm{M} / \mathrm{min}$ ".

Problem: $\left.N T\right|_{C S}<\left.N T\right|_{C T}$ $S V S=\left(0, \delta\left(\left.N T\right|_{C T},\left.N T\right|_{C S}\right)\right)$

Network traffic broker: a service which can firstly take the request from the customer side of "100M/Minute", secondarily separate the request into two parallel tasks and finally distribute the two tasks for two services of "band width of 50M/Minute ", can play the broker.

Solution: $\sum\left(\left.R A\right|_{C S}=\left.R A\right|_{i S V B}\right) A N D\left(\left.R A\right|_{o S V B}\right.$ $\left.>=\left.R A\right|_{C T}\right)$

v. Price $\left(P R \in \mathbb{D}_{B}\right)$ : price of the service usage is put as "10-20 USD/month for USA users" while the customer want "5-10 USD/ month for Asia user". Problem: $\left.P R\right|_{C S}>\left.P R\right|_{C T}$ $S V S=\left(0, \delta\left(\left.P R\right|_{C T},\left.P R\right|_{C S}\right)\right)$

Price broker: the price broker is implemented with flexible strategies such as asking a location broker to convey the request of USA from USA to Asia. If the final price after subtracting the cost of introducing location broker is lower than the original price, the location broker actually implements the role of price broker. There will be other forms of price broker which depends on the specific constraints of the service contracts of both the request and the answer sides. Solution: $\left(\left.P R\right|_{C S}=\left.P R\right|_{i S V B}\right) A N D\left(\left.P R\right|_{o S V B}=\left.P R\right|_{C T}\right)$ vi. Reputation limit $\left(R E \in \mathbb{D}_{S}\right)$ :The service provider is with a reputation grade of " 3 stars" while the customer service demands " 4 stars".

Problem: $\left.R E\right|_{C S}>\left.R E\right|_{C T}$ 
$S V S=\left(0, \delta\left(\left.R E\right|_{C T},\left.R E\right|_{C S}\right)\right)$

Reputation broker: as the reputation of service provider might be based on the rigid data of its historical performance which will be reflect a drastic performance upgrade based on trustworthy technology transfer, etc. A friend service with a strong reputation can be allowed to help to convince the customers with its higher reputation.

Solution: $\left(\left.R E\right|_{C S}<\left.D E(\right.$ task $\left.)\right|_{i S V B}\right) A N D\left(\left.D E\right|_{o S V B}>\right.$ $\left.\left.D E\right|_{C T}\right)$

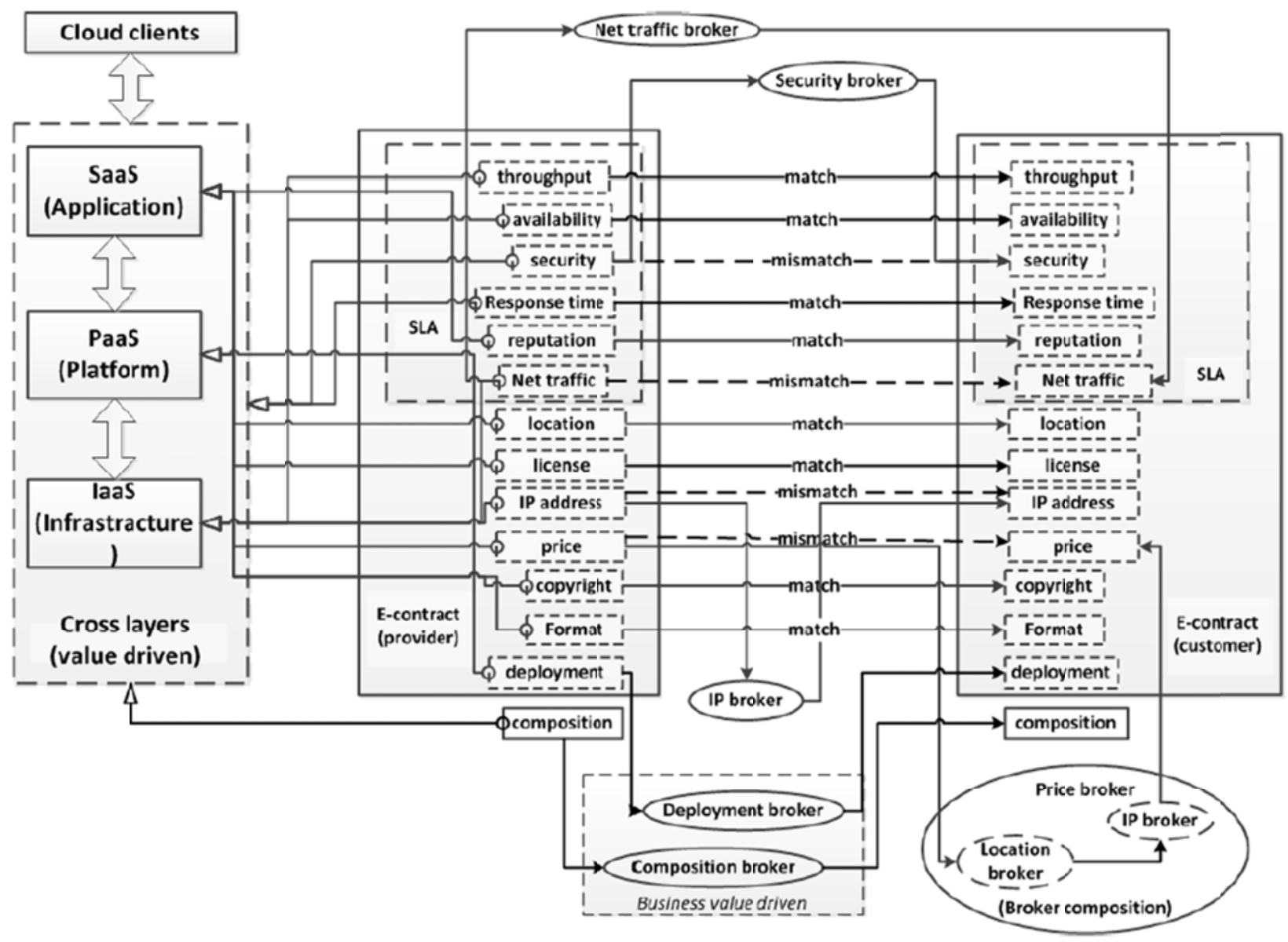

Figure. 1. SVB and DSVB in the context of Cloud

vii. Response time $\left(R T \quad D_{Q}\right)$ : the provided response time is "within 1-2 second/ per process" while the expected response time is " $1-0.5$ second/ per process". Problem: $\left.R T\right|_{C S}>\left.R T\right|_{C T}$ $S V S=\left(0, \delta\left(\left.R T\right|_{C T},\left.R T\right|_{C S}\right)\right)$
Response time broker: a service or a set of services which can play the role in network traffic broker can reduce the response time when the time limit is caused by big amount of data transfer. It can separate the amount of data to be transferred into two tasks and ask 
two instances of the answering/requesting service to send/receive concurrently and combine the data later.

Solution: $\left(\left.R T\right|_{C S}=\left.R T\right|_{i S V B} / \sum\right) A N D\left(\left.R T\right|_{o S V B} / \sum=R T\right.$ $\left.\left.\right|_{C T}\right)$

viii. Currency exchange $\left(C E \mathbb{D}_{B}\right)$ : requested to be paid with " accept Euro for payment" while the customer want to pay with "USD or local money".

Problem: $\left.C E\right|_{C S} !=\left.C E\right|_{C T}$

$S V S=\left(0, \delta\left(\left.C E\right|_{C T},\left.C E\right|_{C S}\right)\right)$

Currency exchange broker: a service which can do real time currency exchange calculation has the possibility of playing the broker.

Solution: $\left(\left.C E\right|_{C S}=\left.C E\right|_{i S V B}\right) A N D\left(\left.C E\right|_{o S V B}=\left.C E\right|_{C T}\right)$

ix. File/data format $\left(F F \quad \mathbb{D}_{B}\right)$ : requested to provide files with "MS word format" while the provider supplies only files with "pdf or ps format".

Problem: $\left.F F\right|_{C S} !=\left.F F\right|_{C T}$

$S V S=\left(0, \delta\left(\left.F F\right|_{C T},\left.F F\right|_{C S}\right)\right)$

File/data format broker: a service which can convert file format from "pdf or ps format" to "MS word format" has the possibility of playing the broker.

Solution: $\left(\left.F F\right|_{C S}=\left.F F\right|_{i S V B}\right) A N D\left(\left.F F\right|_{o S V B}=\left.F F\right|_{C T}\right)$

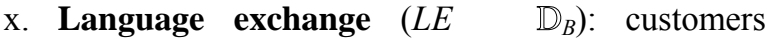
provides information or even E-Contracts in English or Html while the provider accepts only French language or XML, and the negotiation information is blocked.

Problem: $\left.L E\right|_{C S} !=\left.L E\right|_{C T}$

$S V S=\left(0, \delta\left(\left.L E\right|_{C T},\left.L E\right|_{C S}\right)\right)$

File/data format broker: a service which can convert language format from "English/html" to "French/XML"in a bidirectional manner has the possibility of playing the broker.

Solution: $\left(\left.L E\right|_{C S}=\left.L E\right|_{i S V B}\right) A N D\left(\left.L E\right|_{o S V B}=\left.L E\right|_{C T}\right)$

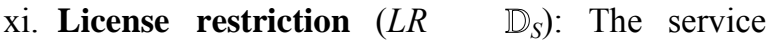
Security broker: a distributed mode of pubic-private key architecture can be introduced to enhancing security level of the provided service while not break the integrity of the original service. For example, the introduction of audition service and a keying system, can help to avoid a denial-of-service attack (DoS) on the main service.

Solution: $\left(\left.S L\right|_{C S}=\left.S L\right|_{i S V B}\right) A N D\left(\left.S L\right|_{o S V B}=\left.S L\right|_{C T}\right)$

xiv.Privacy protection $\left(P P \quad \mathbb{D}_{S}\right)$ : Both providers' provider put a license restriction on that only authorized user can access the function after payment or only requests from registered IP address can be processed.

Problem: $\left.L R\right|_{C S} !=\left.L R\right|_{C T}$

$S V S=\left(0, \delta\left(\left.L R\right|_{C T},\left.L R\right|_{C S}\right)\right)$

License broker: the implementation of this broker is highly flexible. If it is a IP restriction, the previous IP broker can also work as a solution by using a registered IP address to transferring the original requests if transferring requests is not forbidden. If the payment is too high to be accepted by the customer, a service which has paid for the function can help by selling the function to the customer with a pay by use business mode. There will be a lot of law related issues which bring both new solutions and new challenges [9].

Solution: $\left(\left.L R\right|_{C S}=\left.L R\right|_{i S V B}\right) A N D\left(\left.L R\right|_{o S V B}=\left.L R\right|_{C T}\right)$

xii. Storage quantity limit $\left(S Q \quad \mathbb{D}_{F}\right)$ : The service provider assigns a maximum storage capacity of "50M" while the customer service need "1000M or more".

Problem: $\left.S Q\right|_{C S}>\left.S Q\right|_{C T}$

$S V S=\left(0, \delta\left(\left.S Q\right|_{C T},\left.S Q\right|_{C S}\right)\right)$

Storage broker: The combination of a cloud storage service plus a IP broker SVB can fill this gap of requirement.

Solution: $\left(\left.S Q\right|_{C S}<=\left.\sum_{I P} S Q\right|_{i S V B}\right) A N D\left(\left.\sum_{I P} S Q\right|_{o S V B}>=\right.$ $\left.\left.S Q\right|_{C T}\right)$

xiii. Security limit $\left(S L \mathbb{D}_{S}\right)$ : there will be many restrictions of security which might be difficult for a functional service which is not devoted to security to fulfill the task.

Problem: $\left.S L\right|_{C S} !=\left.S L\right|_{C T}$

$S V S=\left(0, \delta\left(\left.S L\right|_{C T},\left.S L\right|_{C S}\right)\right)$

and customers' sides may want to keep their identity information, transaction information, purchase habit, etc., limited to the transaction purpose, either from a business competition consideration or from the individual concerns.

Problem: $\left.P P\right|_{C S}=\left.P P\right|_{C T}$

$S V S=\left(0, \delta\left(\left.P P\right|_{C T},\left.P P\right|_{C S}\right)\right)$

Privacy broker: in general, brokers may play the role in representing the customer/provider to implement a 
transaction indirectly. Then they have the chance to separate the information from being traced back to the actually involved customer or provider by keeping the information security.

$$
\begin{aligned}
& \text { Solution: }\left(\left.P P\right|_{C S}=\left.P P\right|_{i S V B(a)}\right) \quad A N D \quad\left(\left.P P\right|_{o S V B(a)}=\right. \\
& \left.\left.P P\right|_{i S V B(b)}\right) A N D\left(\left.P P\right|_{o S V B(b)}=\left.P P\right|_{C T}\right)
\end{aligned}
$$

\section{Value broker}

Value broker or DSVB is a general form of price broker. It is different from previous brokers which is introduced to solve a mismatch of the conditions of composition which is demanded rigidly by a service matchmaking process [9]. Value broker is introduced as a means of implementation of the optimization process which pursues a better business profit for the stakeholder who employs the service based transaction. A glance of value broker enabled maximization of business solution space is shown in Figure.2 [11]. Theoretically during the implementation of a DSVB, all possibilities of service compositions should be considered including those situations where service composition are enabled by SVB.

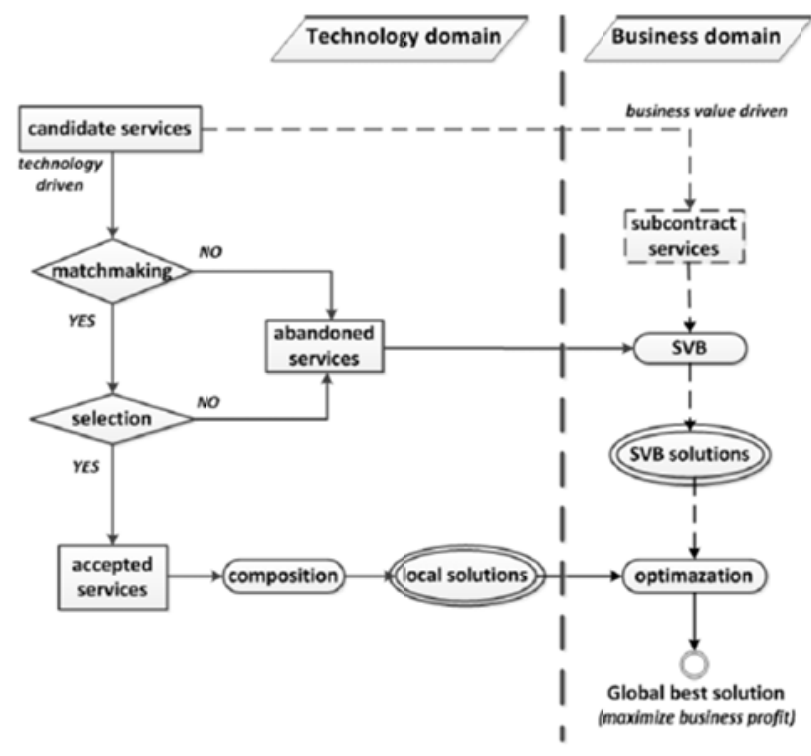

Figure. 2. SVB enabled maximization of business profit space

i. Deployment efficiency $\left(D E \quad \mathbb{D}_{F}\right)$ : in E-Service economics, providers of E-Services will be specialized with accumulated knowledge and reusable experience. A functional service can save the cost and improve the efficiency by packaging a deployment job to a specialized broker service.

Target: $\left.D E\right|_{C S}<\left.D E\right|_{S V B}$

$S V S=\left(0, \delta\left(\left.D E\right|_{S V B},\left.D E\right|_{C S}\right)\right)$

Deployment broker: a customer service need only to describe the requirement on deployment, negotiate the interface and leave the deployment task to the broker.

Solution: $\left.\left.D E($ task $)\right|_{C S} \rightarrow D E($ task $)\right|_{S V B}$

ii. Test efficiency $\left(T E \quad D_{F}\right)$ : by employing a professional service which is devoted to test as a broker, test efficiency and correctness can be expected to be improved, and the cost can be reduced.

Target: $\left.T E\right|_{C S}<\left.D E\right|_{S V B}$

$S V S=\left(0, \delta\left(\left.T E\right|_{S V B},\left.T E\right|_{C S}\right)\right)$

Test broker:a customer service need only to describe the requirement on test, negotiate the interface and leave the test task to the broker which reuses its experience to finish the work with lower cost on learning.

Solution: $\left.\left.T E($ task $)\right|_{C S} \rightarrow T E($ task $)\right|_{S V B}$

iii. Composition optimization $\left(\mathrm{CO} \quad \mathbb{D}_{Q}\right)$ : by employing a professional service which is devoted to composition analysis, a better comprehensive optimization can be expected with lower cost.

Target: $\left.C O\right|_{C S}<\left.C O\right|_{S V B}$

$S V S=\left(0, \delta\left(\left.C O\right|_{S V B} ;\left.C O\right|_{C S}\right)\right)$

Composition broker: the manner of composition might influence the performance of a service greatly. For example, sequentially a service chain's throughput might be restricted by the one with the lowest throughput while a parallelization might save the situation, and for another situation, a decentralization can help to meet the restriction on the amount of input services.

Solution: $\left.\left.\mathrm{CO}($ task $)\right|_{C S} \rightarrow C O($ task $)\right|_{S V B}$

iv. Advertisement efficiency $\left(A E \quad \mathbb{D}_{B}\right)$ : at business application level, advertisement can be trusted to a professional advertisement service.

Target: $\left.A E\right|_{C S}<\left.A E\right|_{C T}$

$S V S=\left(0, \delta\left(\left.A E\right|_{C T},\left.A E\right|_{C S}\right)\right)$

Advertisement broker: by subcontracting an advertisement to a professional advertisement service, it actually implement a reuse of resources including knowledge, etc.

Solution: $\left.\left.A E($ task $)\right|_{C S} \rightarrow A E($ task $)\right|_{S V B}$

v. Auditing efficiency $\left(A E 2 \quad \mathbb{D}_{B}\right)$ : at business 
application level, auditing can be trusted to a professional auditing service.

Target: $\left.A E 2\right|_{C S}<\left.A E 2\right|_{C T}$

$S V S=\left(0, \delta\left(\left.A E 2\right|_{C T} ;\left.A E 2\right|_{C S}\right)\right)$

Auditing broker: by subcontracting the auditing to a professional service, it actually implement a reuse of resources including professional auditing knowledge and save the cost drastically in comparison to maintain a private team for middle sized companies, etc.

Solution: AE2(task) $\left.\right|_{C S} \rightarrow$ AE2(task) $\left.\right|_{S V B}$

numerous application level brokers such as: vender broker, data cleaning broker, etc.

Solution: $\left.\left.W F($ task $)\right|_{C S} \rightarrow W F($ task $)\right|_{S V B}$

vii. Reuse development $\left(R D \quad \mathbb{D}_{B}\right)$ : reuse of service execution or service data or service information can bring the increase of business profit for both customer and provider sides.

Target: $\left.R D\right|_{C T} \rightarrow \operatorname{new}\left(\left.R D\right|_{C S}\right)$

$S V S=\left(0, \delta\left(\left.R D\right|_{C T},\left.R D\right|_{C S}\right)\right)$

Reuse broker: reuse of service execution can happen when the part or the whole information which is generated by a service execution can be shared by other customers, or the data which is generated by a service execution or a group of services can be collected and be of value to another usages. A service which manage the strategy discussed above can play the broker.

Solution: $\left.R D\right|_{C S} \rightarrow$ share(information) $\left.\right|_{\text {new (CS) }}$ ORRD $\left.\right|_{C S}$ $\rightarrow$ information $\left.\right|_{\text {new }(C S)}$

viii. Risk insurance $\left(R I \quad D_{B}\right)$ : risks of a transaction can be avoided at implementation level and economical level. Here we refer to economical means to transfer risks.

Target: $\left.\left.R I\right|_{C S} \rightarrow \infty O R R I\right|_{C T} \rightarrow \infty$

$S V S=\left(0, \delta\left(\left.R I\right|_{C T},\left.R I\right|_{C S}\right)\right)$

Risk broker: economically insurance services can be introduced as brokers for the transaction either on the customer side or the provider side or on the transaction.

Solution: $\quad\left(\left.R I\right|_{C S} \rightarrow\right.$ Insurence $\left.\left.\right|_{C S}\right)$ OR $\left(\left.R I\right|_{C T} \quad \rightarrow\right.$ Insurence $\left.\left.\right|_{C T}\right)$

ix. Health care $\left(H C \mathbb{D}_{B} \cap \mathbb{D}_{F}\right)$ : the diagnosis, treatment, and prevention of disease and the choosing of medicine and treatment needs a tradeoff between the functional effects and economical feasibility. Knowledge from clinical doctors and economical advisors will be combined before a decision on personal resources is made. vi. Weather forecasting $\left(W F \quad \mathbb{D}_{B}\right)$ : weather forecast is a costly and challenging task, however a lot of organizations might need this service with specific precision request.

Target: $\left.W F\right|_{C S}<\left.W F\right|_{C T}$

$S V S=\left(0, \delta\left(\left.W F\right|_{C T},\left.W F\right|_{C S}\right)\right)$

weather forecasting broker: by subcontracting the weather forecasting to a professional service, it actually implement a reuse of resources including professional knowledge, etc. Similarly we can identify Target: optimization $\left(\left.\operatorname{cost}\right|_{C S} \rightarrow \infty\right.$ ORlife $\left.\left.\right|_{C S} \rightarrow 0\right)$

$S V S=\left(0, \delta\left(\left.H C\right|_{C T},\left.H C\right|_{C S}\right)\right)$

Health care broker: constructing a health care, a broker needs a balance on the available resources on personal side, the individual health conditions, medical knowledge and cost of treatment, etc. It will involve knowledge pooling and moral consideration.

Solution: $\left.\mathrm{HC}\right|_{C S} \rightarrow$ balance(health $\left.\right|_{C S} A N D$ resource $\left.\right|_{C S}$ AND $\left.\left.\operatorname{cost}\right|_{C T}\right)$

Tradeoff of cost vs. gain: a value driven optimization should pursue a balance of the ratio between the cost of traversing all possibilities and the expected profit of the transaction. Further work should be devoted to many forms of value which are difficult or impossible to be normalized and quantified in terms of monetary value including location restriction, policy restriction, time, life, moral, reputation and trust,[10], [8], [16] during a value driven service transaction. A glance of the scenario of SVB and DSVB in the context of service contract including SLA can be can be found in Figure.1.

\section{Composition and Organization of SVB}

\subsection{The general business scenario}

Figure 3 shows the general scenario of multiple service values from mainly three sources. We summarize them as follows:

i. Provider value $(P R V)$ - At the service provider side, business value needs to be considered from the temporal dimension as short run vs. long run target which will decide specific business strategies such as new product advertisement, promotion, sell out, etc. Among providers the value can be classified into two categories: 
a. Negative competitive cost - Negative competitive cost occurs when other business b. Positive cooperative wins - When service vendors who offer related or similar services agree on some fixed conditions such as market share, sells area, etc, they can build some cooperations to profit from the customer side such as lifting the price of services or charges of maintenance, etc.

ii. Customer value (CSV) - Service customers in general have independent views on the value of the targeted services. However customers can socialize with other customers to query the quality of a service from others' experiences and comments. The experience information or news/advertisement propagated through social media among customers is playing an increasing role in promoting sales and adjusting commerce behavior. Customers can also build federations to protect their shared interests against malicious service providers with shared cost. Small scale of customer cooperation can cooperate to win promotion sale packages from providers in a winwin manner.

iii. Public value (PUV) - The public administration is the third party which can play the juridical role for solving the argumentation. The public administration also has other critical responsibilities: (i) monitor the service market through economical analysis to avoid the competition between the provider and customer side to enter an Zero-Sum game; (ii) employ public policies to intervene the strong cooperation against customer interests at the provider side, or collusive customers [37], etc.

\subsection{Composition of SVB}

There are various situations where SVBs are composed with different cardinalities of " $1: 1 "$, " $1: n "$, "m:n", and sequences. We classify the composition modes as follows: competitors who offer similar services bid for the same order or market.

i. Vertical composition: We take the Location broker as an example.If requests are restricted to be "from China" while the customer want to visit "as much nations of Europe Union as possible" without additional registration. Then a solution can be built on the integration of Location broker which takes request "from China" while has the authority to issue the pass for only one nation.

Solution: $\left(\left.L C\right|_{C S}=\left\{\left.L C\right|_{i S V B}\right\}_{1 \ldots n}\right) A N D\left(\left\{\left.L C\right|_{o S V B}\right\}_{1 \ldots n}=\right.$ $\left.\left.L C\right|_{C T}\right) A N D\left(\sum\left(\right.\right.$ coverage $\left.\left.(L C)\right|_{\text {oSVB }}\right)=$ coverage $\left.\left.\right|_{C T}\right)$

ii. Horizontal composition: We take the Currency broker as an example. The payment is restricted to be "Czech Koruna" while the customer has only "Thai Baht". If a Currency exchange broker (a) which exchange "Thai Baht" to "Euro", and a Currency exchange broker (b) which exchange "Euro" to "Czech Koruna" are available. The connection of the two brokers will construct a solution from this customer to the provider.

Solution: $\left(\left.C E\right|_{C S}=\left.C E\right|_{i S V B(a)}\right) A N D\left(\left.C E\right|_{o S V B(a)}=\right.$ $\left.\left.C E\right|_{i S V B(b)}\right) A N D\left(\left.C E\right|_{o S V B(b)}=\left.C E\right|_{C T}\right)$

iii. Intelligent composition: For an agent SVB [30], to increase its general profit for providers it will consider the provider side situation such as the real time sales data and yearly historical sales record at different seasons. After a comprehensive calculation, it will offer probably a discount strategy or advertisement plan, such as a case that if a customer can purchase several services as a bundle, there will be a 30 percent discount which will be mutual beneficial to both customers and the provider. Also at customer side, if negotiation is permitted among customers, they can form Service federations to jointly collect and build their purchase items into service bundles to benefit from the discount offer from the provider side. 


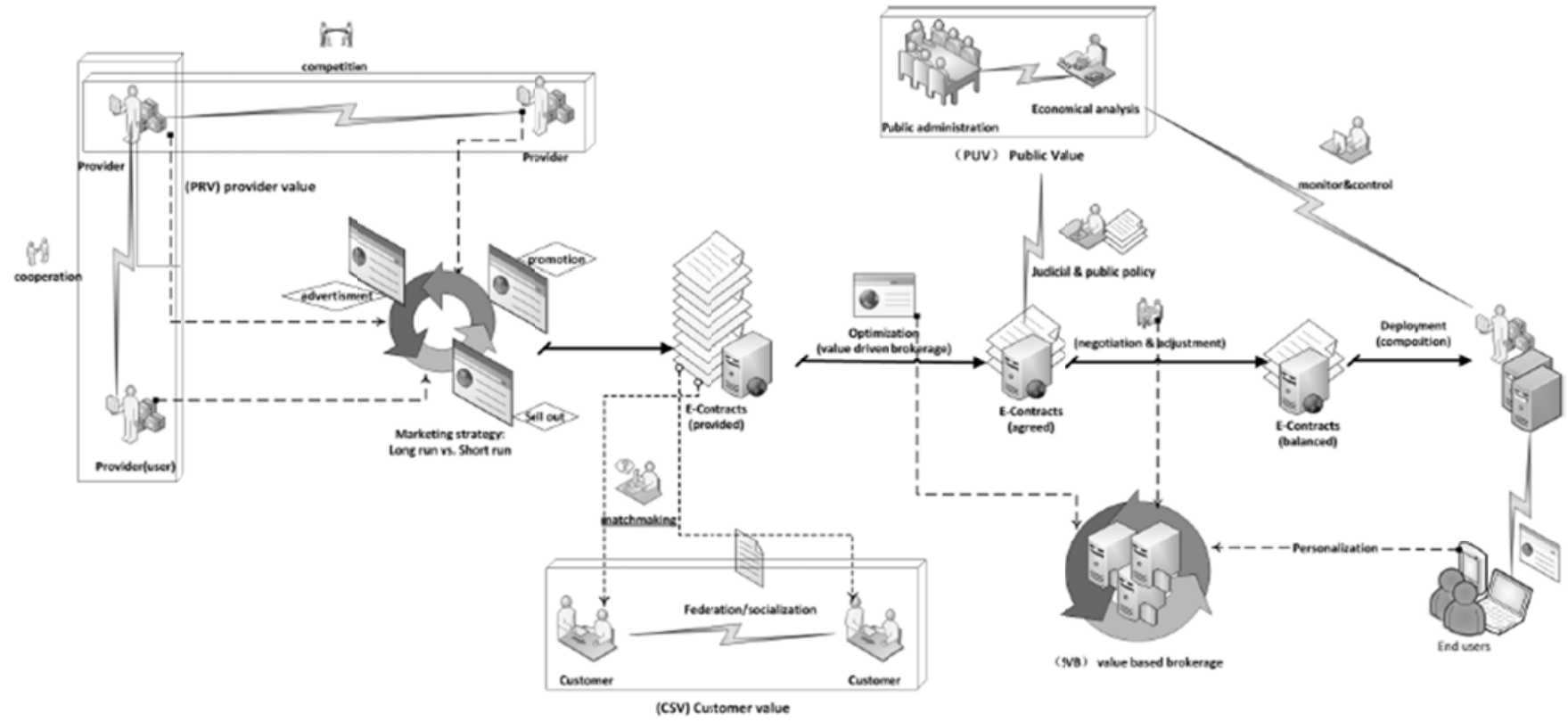

Figure. 3. Integrating value considerations from multiple stakeholder with value brokerage

\subsection{Broker refinement}

Many situations can be refined for above mentioned basic brokers. We extend the discussion on Security broker as

Follows.

i. Operation security: $\left(O S \mathbb{D}_{S}\right)$ the completeness and reliability of a series of operations and behaviors of a service transaction is defined as operation security.

Operation security broker: a service which checks the atomic actions and monitors implementation of the sequence of execution or protocol of interactions can play the broker.

Solution: $\left(\left.O S\right|_{C S} \rightarrow\right.$ check $\left.($ atomic $\left.)\right|_{i S V B}\right)$ AND $\left(\left.O S\right|_{C T} \rightarrow\right.$ monitor(protocol $\left.\left.)\left.\right|_{o S V B}\right)\right)$

ii. Data security: $\left(D S \quad \mathbb{D}_{S}\right)$ the integrity, correctness and completeness of the data of a service transaction is defined as data security.

Data security broker: a service which controls the access of the data, maintains the completeness and checks the integraty of service data can play the broker. The implementation can be through encryption broker, etc.

Solution: $\quad\left(\left.D S\right|_{C S}\right.$ control(access $)\left.\right|_{\text {iSVBANDoSVB }} A N D\left(\left.D S\right|_{C S}\right.$ maintain(completeness) $\left.\left.\right|_{i S V B}\right) A N D\left(\left.D S\right|_{C T}\right.$ check(integraty) $\left.\left.\right|_{\text {osvB }}\right)$ )

We extend the discussion on Privacy broker as follows.

i. Operation privacy: $\left(O P \quad D_{S}\right)$ the operations and behaviors of a customer can be used to identify the personal information which the customer may not want to disclose. The habit of a customer which is embodied in the historical purchase records can be used by the provider or its competitor to make either positively or negatively customized business strategies. Also the behavior record of a provider can be used for similar unwanted purpose.

Operation privacy broker: a lot of strategies can be practiced to cope this situations, such as (a) several services can build a federation to mix their behaviors to increase the difficulty of analyzing their information, (b) Location broker and IP broker can be used to switch the traces of a customer/provider's transaction space, and (c) Available time broker can be used to break the rhythm analysis against a customer/provider's transaction time .

Solution: $\left(\left.O P\right|_{O S} \rightarrow L C,\left.A T\right|_{i S V B}\right) A N D\left(\left.O P\right|_{C T} \rightarrow\right.$ $\left.\operatorname{break}($ tracking $\left.)\right|_{\text {oSVB }}\right)$ )

ii. Information privacy:(IP $\left.\quad \mathbb{D}_{S}\right)$ during a 
transaction, some pieces of information which are not required or are not necessary for a transaction might be required or leaked without notice.

Information privacy broker: a service which checks and restricts the usages of service information based on a necessary-only policy may play the broker.

Solution: $\left.\quad\left(\left.I P\right|_{C S} \rightarrow\right.$ (check(access $)\right|_{i S V B} \quad A N D$ validate(necessary) $\left.\left.\right|_{i s V B}\right)$ )

\subsection{Classification of SVB from knowledge management perspective The Classification of $S V B$ is a prerequisite for avoiding}

the cost of reinvent of existing SVB and select the most appropriate ones for specific usage. It is also required for identifying new opportunities of creating new SVB. However this is also a big challenge since it involves knowledge from multiple domains crossing various abstraction layers. The modeling and expression of organization may be very complicated and crosscut multiple interrelated conceptual dimensions/perspectives. In Figure 4 we demonstrate our work towards organizing empirically collected SVB from a knowledge manage perspective. The problem solving framework is composed of three top level categories. At the category of Problem solving, problem to solution is bridged by Knowledge broker. knowledge broker consists the category Resource and the category Implementation. Some previous described SVBs are classified by relating to the items of the categories, such as:

i. Inside the category Resource, information is bridged by translation broker and proxy brokers such as reputation broker, location broker, IP broker, etc.; data is bridged by format broker; operation is refined as control and execution which are bridged or optimized by brokers such as security broker, privacy broker, available time broker, latency broker and throughput broker, etc. It mentions that data might also require security broker and privacy broker according to data contract [35]. It differs from the security broker and privacy broker for operation in the E-Service contract.

ii. The category Implementation is modeled as from description to implementation. The involved activities can be abstracted as decomposition and integration which can be optimized by DSVBs of composition broker and federation broker, etc.

From the domain of E-Tourism, we have identified many application areas which can be implemented with SVB in different categories[14] which is shown in Figure 5. 


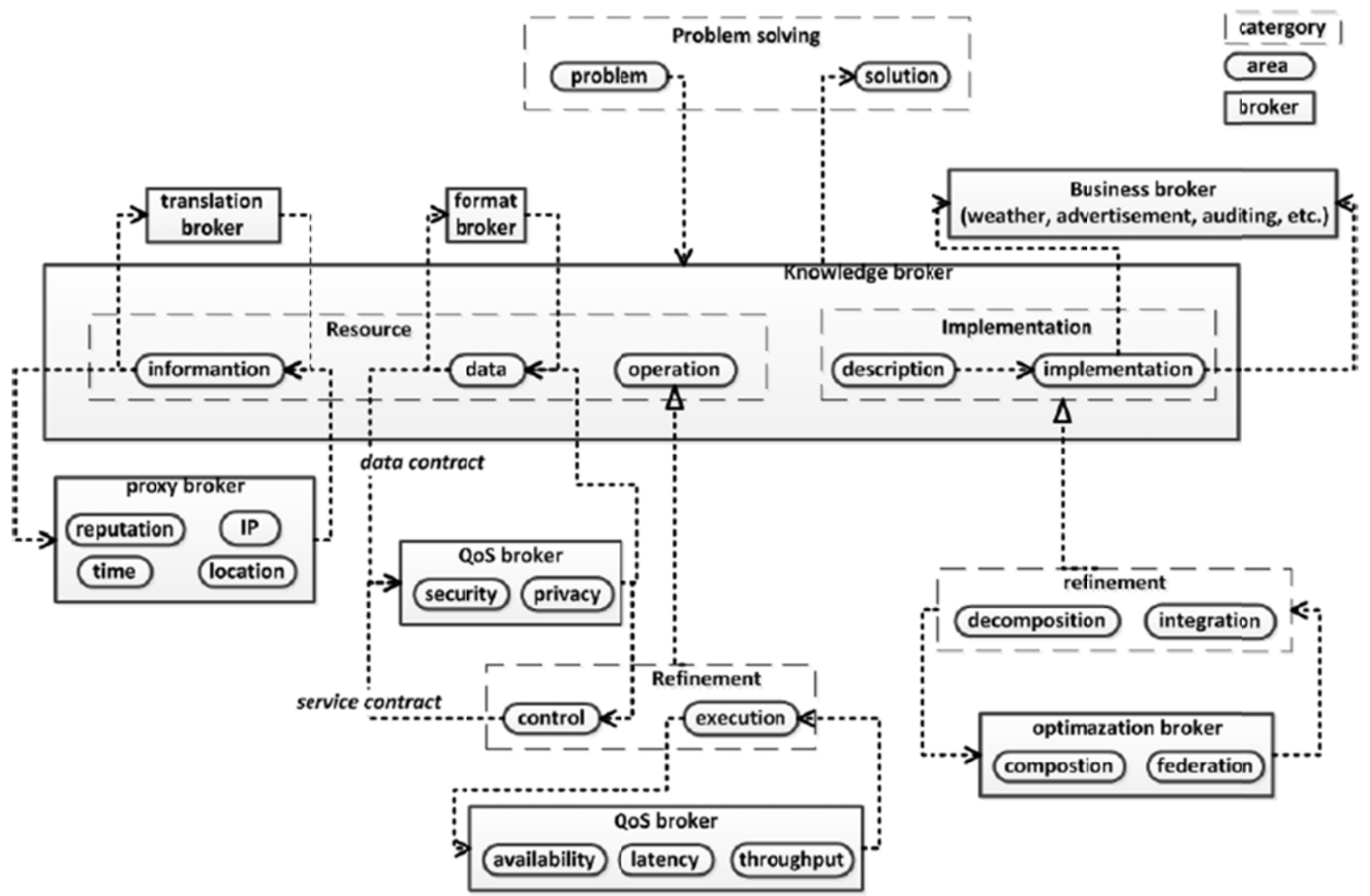

Figure. 4. Classification of SVB from the perspective of knowledge management

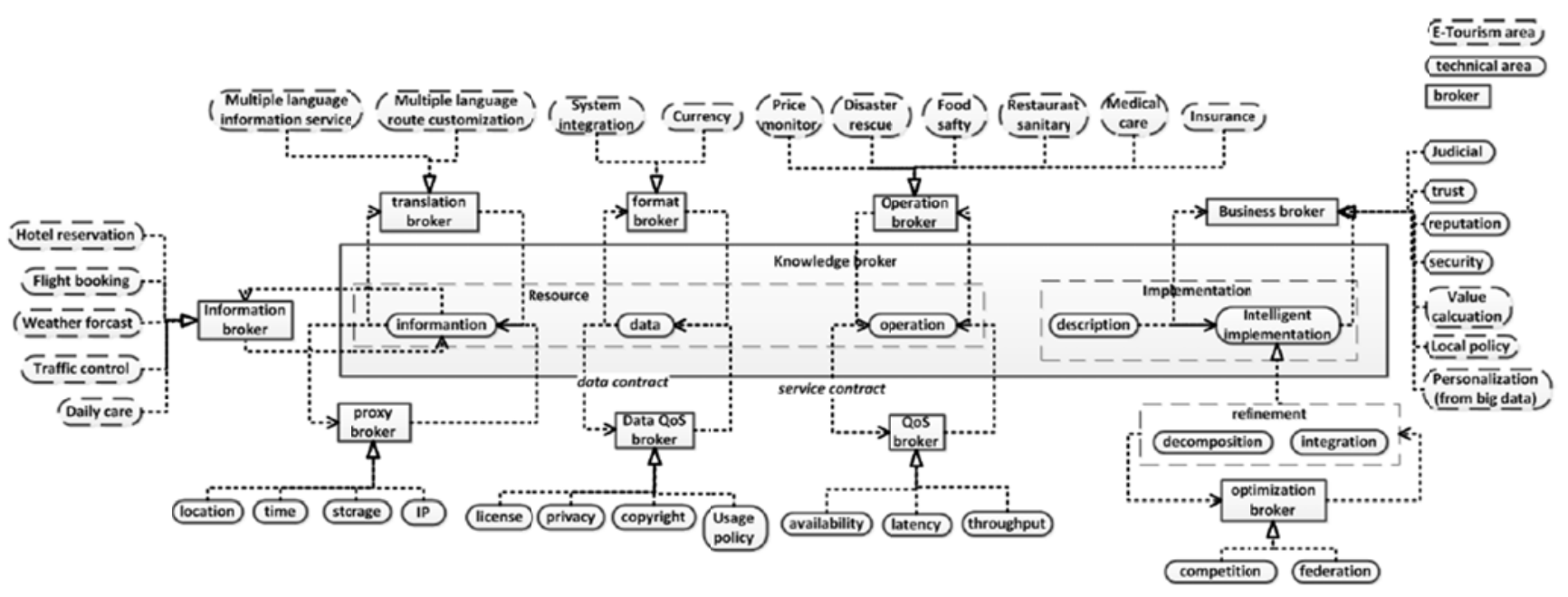

Figure. 5. Empirical SVB classification from an E-tourism background 


\section{Two-Level E-Contract Based Implementation Framework}

In the service ecosystem, due to the interface of the services and their correlation history, the services in the ecosystem will form the composable relation between each other which means that the two services can be used to form a composition to offer added-value for the consumers. As the number of services available for consumers is increasing rapidly, there are many services which offer the similar functionality. For examples,all of "Google Map", "Baidu Map", "Yahoo Map" and "Facebook Map" provide the map related services. These services with the similar functionality will form a specific domain. The service in the same domain can somehow replace each other with some adapters [34]. Furthermore, the providers will publish services into the ecosystem so that the consumers can use the services to fulfill their requirement. Some providers such as Google, Yahoo and Amazon will offer different services in different domains so that they may offer the complete solution for the consumers. Some others will provide a few specific services in the specific domain. Taking Twilio as an example, it focuses on telephony and only offers the Twilio service in the telephony domain for the consumers. As different providers perform well in different domain, the providers will assign the contract with the others to form a vertical alliance or horizontal alliance to guarantee their core competencies [20]: the providers who provide similar services may assign contracts with each other so that they can get the replace services to increase the faulttolerance for the consumers; the providers who provide the composable services may assign contracts with each other that they can increase the Qos for the whole composition.

Thus we can get a two-level service contract framework in the service ecosystem which consists of two networks: the service composable network is a directed network in which each node refers to a service and each edge refers to the composability between two services, the direction of the edge refers that the output of the source service can be the input or part of the input for the target service. The provider contract network is an undirected network in which each node refers to a provider and each edge refers to the service contract assigned by two providers.
Fig. 6 demonstrates a two-level service contract network framework for the service ecosystem which consists two networks: the service composable network which refers to the composablity among services, and the provider contract network which refers to the contract relation among providers.

Example: For the illustration shown in Fig. 6 , providers $\mathrm{Pa}, \mathrm{Pb}, \mathrm{Pc}, \mathrm{Pd}, \mathrm{Pe}$ form the provider contract network based on their contract with each other. Provider Pa offers service $\mathrm{S} 1$ and $\mathrm{S} 2$, Provider Pb offers services S3, S4 and S5, etc. Service s1, s2, s3, s4, s5, s6, s7, s8 and s9 construct the service composable network and S1, s3, and s6 are similar in the functionality that they form a specific domain.

\section{The Case for the Service Contract Broker}

\subsection{Service Contract Broker for Service Selection}

The requirement of the consumer is becoming more complex. Sometime single services cannot fulfill the requirement that they need to select some services to form compositions. If the services are provided by different providers, the providers with a contract can help to guarantee the reputation of the composition. For example, $\mathrm{Pd}$ and $\mathrm{Pc}$ have a contract while it is not for $\mathrm{Pd}$ and $\mathrm{Pb}$, the composition for $\mathrm{s} 6$ and $\mathrm{s} 7$ will gain a higher reputation than s6 and s5. In this case, the service contract broker will suggest services with higher reputation for the consumers. Even if the services are provided by the same provider, sometimes the QoS cannot meet the consumers' requirement. For example, s1 and s2 can fulfill the consumer's functionality requirement while

the price is too high for the consumer. In this case, the service contract broker will help to find the services which are offered by the provider's contractors and then use the service to replace the similar service to fix the mismatch for the consumers. For example, suppose that $\mathrm{s} 3$ is much cheaper than $\mathrm{s} 1$ and then the broker will use s3 to replace s1 and offer $\mathrm{s} 3$ and $\mathrm{s} 2$ for the consumers. 


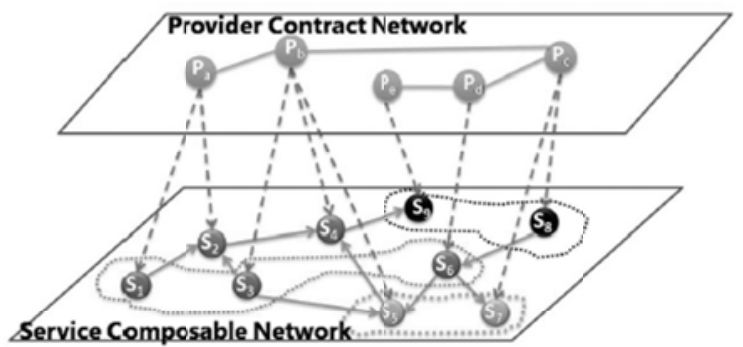

Provider Contract Relation $\longrightarrow$ Service Composable Relation $--\rightarrow$ Provider supply service

Figure. 6. Two-level service contract network framework

\subsection{Service Contract Broker for Service Emerging}

For the providers with a strong contract, if the services they offer are not composable, the providers will intend to build an adapter among their services so that their alliance can gain higher competitiveness in the ecosystem. For example, $\mathrm{Pb}$ offers service $\mathrm{s} 4$ which can be composed with $\mathrm{s} 9$ provided by $\mathrm{Pe}$ and there is no contract between these two providers. Also Pc offers service $\mathrm{s} 8$ which has a similar functionality as $\mathrm{s} 9$, however there is huge mismatch between $\mathrm{s} 4$ and $\mathrm{s} 8$. As $\mathrm{Pb}$ and $\mathrm{Pc}$ builds a strong contract relation with each other, they may modify the interface of their services to make them composable or create a new service together to bridge their services. In this case, the service contract broker will offer the suggestion for new services. Thus the service contract broker can promote the growth of the service ecosystem.

\section{Simulating $S V B$}

In order to simulate $S V B$ patterns and their effect on customer value, we make use of the scenario provided in Fig. 7. While Customer 1 accesses the sequence of services directly, Customer 2 makes use of SVB brokers to aid in his response. This may come from multiple domains of values.

This scenario is simulated using Monte-Carlo simulations in MATLAB with distributions representing various domains of functional, QoS and business value aspects studied in Section II. Values such as response time and availability are modeled as heavy tailed distributions [21]. Request amount and Network Traffic are modeled with exponential distributions; Price, License Values and Security Levels are drawn from uniform distributions. Note that some brokers such as Location and Reputation Limit would require a real-world implementation over actual services and are exempted from this analysis. Such a probabilistic model for value is consistent with perspectives of function/QoS/business [21], [23].

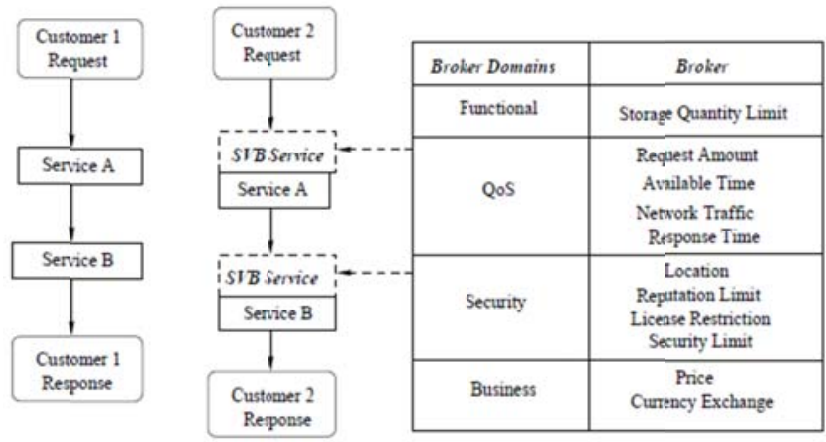

Figure. 7. Scenario comparing two customers.

As observed in Fig. 8, the inclusion of an $S V B$ broker improves multiple domains. The response time distribution and network traffic show lower values for customer 2 . This is traded off with the necessity to pay higher cost values that can provide better security and license values. Though this is a representative example, it can be envisioned as being applicable to real world applications. The service broker can provide access to valuable upgrades in multiple domains that should be encouraged.

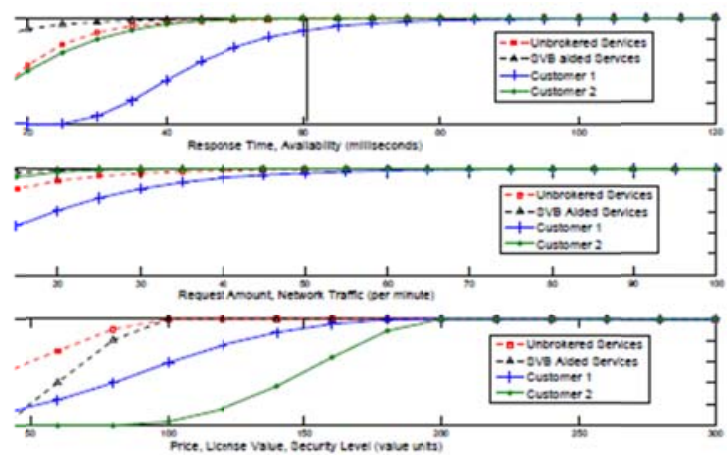

Figure. 8. Monte-Carlo runs of two customers' output behaviors. 
From a business perspective, the improved performance due to the introduction of a broker could provide better contractual agreements to a composition of these services. In spite of higher costing services, the tradeoffs can be improved in multiple contractual domains of QoS, security and composition efficiency. Aspects provided by the DSVB such as testing and advertisement provide further impetus to the adoption of brokers for business based services.

\section{Related Work}

Bichler et al.[1] promote to use brokers to enhance the application level interpretability of electronic commerce. $\mathrm{Yu}$ and Lin[39]utilize service brokers to meet SLAs of services and construct trust network for bridging reputation information[25]. It does not directly support the construction of $\mathbb{D}_{\mathrm{B}}$ and $\mathbb{D}_{\mathrm{V}}$ oriented solutions. Srikumar et al.[36] use a broker to enable grid resource searching and distribution where a broker functions mostly as an autonomous agent[30]. D'Mello et al.[7] use a broker to select qualified services in terms of QoS of SLA for service composition. Loreto et al.[26] use brokers to integrate telephone business and IT world in the manner of a intermediate layer. Most of existing broker researches[28], [24], [31], [4], [27], [17] focus on using brokers to discover, match,negotiate, select and compose services with best QoS in a service composition from either a technological perspective or a business perspective. Rosenberg and Dustdar[33] use brokers to bridge the difference of heterogenous business rules. Budgen et al.[2] introduce an information broker to integrate health knowledge and data with enhanced privacy protection. Based on service contracts, SVB covers more issues than SLA. SVB relates services not limited to technological level as most SLAs based approaches[39] have done but also to business level[1], [33], [3]. Cardellini et al.[3] use brokers to realize a global cost optimization based on probabilities. By integrating business services and technology services with

\section{Acknowledgment}

This paper was supported in part by CNSF grant 61162010 and 61363007 and by HNU Research program value modeling, SVB identifies a bigger diagram where it can be applied positively.

\section{Conclusion and Future Work}

Service value broker (SVB) is a critical element for constructing a coming era of E-Service Economics since it coherently supports IT implementation of service system and integration of business strategies under the analysis of economical goals. The study of SVB by itself will open a lot of research directions:

i. Conceptual level: the introduction of SVB will bring changes to existing architecture of service system;

ii. Solution extension: existing solutions can be extended to this concept such as reuse on SVB level and reuse [11] solutions covering SVB;

iii. Implementation level:SVB will bring influence directly to the service contract description and contract interface design;

iv. Formal abstraction: SVB will be a new target for formal modeling, verification and checking on properties such as equivalence, deadlock[5] and crash situations, etc.

In this paper we present the work towards enumerating useful SVBs which can be reused directly by stakeholders. We would like to explore higher level SVB or DSVB which might be in the form of semantics brokers with contextual information such as temporal constraint [6], reputation network [38] and service evolution [32], etc. Like a blade with two edges, the usages of SVB could introduce new challenges such as fraud SVB, loose controllability due to indirectly control, shattered responsibilities, etc. We are interested in exploring the dimension of avoiding the negative usages of SVB through restricting the possibilities of unexpected subcontract relationships, monitoring the chained behavior and identifying the responsibilities, etc. We would like to see a deepening influence brought by SVB to the era of E-Service Economics[15].

grant KYQD1242 and HDSF201310 . * stands for corresponding author. We thank Prof. Zibin Zheng for precious advice. 


\section{References}

1. M. Bichler, A. Segev, and C. Beam, "An electronic broker for business-to-business electronic commerce on the internet," Int. J. Cooperative Inf. Syst., vol. 7, no. 4, pp. 315-330, 1998.

2. D. Budgen, M. Rigby, P. Brereton, and M. Turner, "A data integration broker for healthcare systems," IEEE Computer, vol. 40, no. 4, pp. 34-41, 2007.

3. V. Cardellini, E. Casalicchio, V. Grassi, and R. Mirandola, “A framework for optimal service selection in broker-based architectures with multiple qos classes," in SCW, 2006, pp. 105-112.

4. F. Casati, S. Ilnicki, L.-j. Jin, V. Krishnamoorthy, and M.-C. Shan, "Adaptive and dynamic service composition in eflow," in CAiSE 2000, pp. 13-31.

5. S. Chen, Y. Deng, P. C. Attie, and W. Sun, "Optimal deadlock detection in distributed systems based on locally constructed wait-for graphs," in ICDCS, 1996, pp. 613-619.

6. T. Cucinotta, G. Anastasi, and L. Abeni, "Respecting temporal constraints in virtualised services," in COMPSAC (2), 2009, pp. 73-78.

7. D. A. D'Mello, V. S. Ananthanarayana, and S. Thilagam, "A qos broker based architecture for dynamic web service selection," in Proceedings of AMS2008, pp. 101-106.

8. Y. Duan, "Catering Quality Evaluation Design for Service/Cloud Computing through Visualized Semantics Locating," SSNE, pp. 45-50, 2011.

9. Y. Duan, "A Survey on Service Contract," in Proceedings of SNPD 2012. IEEE Computer Society, 2012.

10. Y. Duan, "Modeling service value transfer beyond normalization," in SNPD, 2012, pp. 811-816.

11. Y. Duan, "Value Modeling and Calculation for Everything as a Service (XaaS) based on Reuse," in Proceedings of SNPD 2012. IEEE Computer Society, 2012.

12. Y. Duan, C. Cruz, A. Elfaki, Y. Bai, and W. Du, "Modeling value evaluation of semantics aided secondary language acquisition as model driven knowledge management," in IEEE ICIS2013.

13. Y. Duan, A. Kattepur, H. Zhou, Y. Chang, M. Huang, and W. Du, "Service value broker patterns: towards the foundation," in IEEE ICIS2013.

14. Y. Duan, A. Kattepur, H. Zhou, Y. Chang, M. Huang, and W. Du, "Service value broker patterns: An empirical collection," in SNPD2013, July 2013, pp. 675-682.

15. Y. Duan, H. Zhou, Y. Chang, M. Huang, S. Chen, A. Elfaki, and W. Du, "Characterizing e-service economics based on e-contract and driven by e-value," in IEEE ICIS2013.

16. D. J. Dubois, C. Nikolaou, and M. Voskakis, “A Model Transformation for Increasing Value in Service Networks through Intangible Value Exchanges," in Proceedings of ICSS2010. IEEE Computer Society, pp. 185-189.

17. R. Farmer, A. Raybone, R. Uddin, M. Odetayo, and K.M. Chao, "Metadata discovery for a service-broker architecture," in Proceedings of the 2008 IEEE International Conference on e-Business Engineering, pp. 173-178.

18. E. Gamma, R. Helm, R. E. Johnson, and J. M. Vlissides, "Design patterns: Abstraction and reuse of objectoriented design," in ECOOP, 1993, pp. 406-431.

19. J. Gordijn and J. M. Akkermans, "Value-based requirements engineering: exploring innovative ecommerce ideas," Requir. Eng., vol. 8, no. 2, pp. 114134, 2003.

20. K. Huang, Y. Fan, W. Tan, and M. Qian, "Bsnet: a networkbased framework for service-oriented business ecosystem management," Concurrency and Computation: Practice and Experience, vol. 25, no. 13, pp. 1861-1878, 2013.

21. A. Kattepur, "Importance sampling of probabilistic contracts in web services," ser. ICSOC, 2011, pp. 557565.

22. A. Kattepur, A. Benveniste, and C. Jard, "Optimizing decisions in web services orchestrations," in ICSOC, 2011, pp. 77-91.

23. A. Kattepur, A. Benveniste, and C. Jard, "Negotiation strategies for probabilistic contracts in web services orchestrations," in ICWS, 2012, pp. 106-113.

24. A. Kumar.p.s, G. Mahadevan, and G. Krishna.c, "Article: A qos towards dynamic web services recapitulation and selection," International Journal of Computer Applications, vol. 54, no. 4, pp. 12-18, 
September 2012.

25. K.-J. Lin, H. Lu, T. Yu, and C.-e. Tai, "A reputation and trust management broker framework for web applications," in Proceedings of the IEEE EEE, 2005, pp. 262-269.

26. S. Loreto, T. Mecklin, M. Opsenica, and H.-M. Rissanen, "Service broker architecture: location business case and mashups," Comm. Mag., vol. 47, no. 4, pp. 97-103, Apr. 2009.

27. B. Moore and Q. H. Mahmoud, "A service broker and business model for saas applications," in AICCSA, 2009, pp. 322-329.

28. Z. Pan and J. Baik, "Qos broker-based trust model for effective web service selection," in Proceedings of the $11^{\text {th }}$ IASTED SEA2007, Anaheim, CA, USA, pp. 590595.

29. D. Plummer, "Cloud services brokerage: A must-have for most organizations," Gartner, Inc., 2012.

30. Z. Qian, S. Lu, and L. Xie, "Mobile-agent-based web service composition," in 4th Intl. conf. on Grid and Cooperative Computing, pp. 35-46.

31. S. Ran, "A model for web services discovery with qos," SIGecom Exch., vol. 4, no. 1, pp. 1-10, Mar. 2003.

32. D. Romano and M. Pinzger, "Analyzing the evolution of web services using fine-grained changes," in ICWS, 2012, pp. 392-399.

33. F. Rosenberg and S. Dustdar, "Design and implementation of a service-oriented business rules broker," in $C E C W, 2005$, pp. 55-63.

34. W. Tan and M. Zhou, Business and Scientific Workflows: A Web Service-Oriented Approach, ser. IEEE Press Series on Systems Science and Engineering. Wiley, 2013.

35. H. L. Truong, M. Comerio, F. D. Paoli, G. R. Gangadharan, and S. Dustdar, "Data contracts for cloud-based data marketplaces," IJCSE, vol. 7, no. 4, pp. 280-295, 2012.

36. S. Venugopal, R. Buyya, and L. Winton, “A grid service broker for scheduling distributed data-oriented applications on global grids," in $M G C, 2004$, pp. 7580.

37. Y. Wang and J. Wei, "Viaf: Verification-based integrity assurance framework for mapreduce," in IEEE CLOUD, 2011, pp. 300-307.
38. J. Yao, W. Tan, S. Nepal, S. Chen, J. Zhang, D. D. Roure, and C. A. Goble, "Reputationnet: A reputation engine to enhance servicemap by recommending trusted services," in IEEE SCC, 2012, pp. 454-461.

39. T. Yu and K.-J. Lin, "A broker-based framework for qos-aware web service composition," in EEE, 2005, pp. 22-29. 